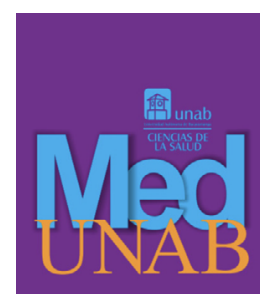

REVISTA DE LA FACULTAD

DE CIENCIAS DE LA SALUD

Vol 23(1):51-61, abril - julio 2020

Artículo original

\title{
Estrategias de formación en valores en estudiantes de un programa técnico de auxiliar de enfermería en Bucaramanga durante el 2018
}

Formation of fundamental values strategies for Students of the assistant nursing technical program in Bucaramanga during 2018

Estratégias de formação de valores para alunos de um curso técnico de auxiliar de enfermagem em Bucaramanga durante o ano de 2018

Rosa Elena Gerardino-Meneses, Enf., Esp., MSc. ${ }^{1}$ (D)

1. Enfermera, Especialista en Docencia Universitaria, Especialista en Seguridad y Salud en el Trabajo, Magíster en Educación con mención en Pedagogía. Docente programa de Enfermería, Universidad Autónoma de Bucaramanga, Santander, Colombia.

Correspondencia. Rosa Elena Gerardino Meneses. Enfermera, Especialista en Docencia Universitaria, Especialista en Seguridad y Salud en el Trabajo, Magíster en Educación con mención en Pedagogía. Programa de Enfermería, Universidad Autónoma de Bucaramanga, Santander, Colombia. Email. rgerardino@unab.edu.co

Cómo citar: Gerardino-Meneses RE. Estrategias de formación en valores en estudiantes de un programa técnico de auxiliar de enfermería en Bucaramanga durante el 2018. MedUNAB. 2020;23(1):51-61. doi: $10.29375 / 01237047.3638$

\section{INFORMACIÓN DEL ARTÍCULO:}

Artículo recibido: 12 de junio de 2019

Artículo aceptado: 11 de febrero de 2020

Doi: https//doi.org/10.29375/01237047.3638

\section{RESUMEN}

Introducción. El cuidado a la salud es una empresa humana, cuyo valor esencial es de índole moral, orientado hacia el bien; entonces, las prácticas de cuidados de salud deberán ser estudiadas y orientadas no sólo por las ciencias naturales, sino también por las ciencias humanas aplicadas. El objetivo del presente artículo es determinar la relación entre las estrategias didácticas de enseñanza y la formación de valores de los estudiantes de primer semestre de un técnico auxiliar en enfermería de una institución de Bucaramanga. Metodología. Corresponde a un estudio cuantitativo, descriptivo correlacional, de corte transversal con una muestra por conveniencia 
de 80 estudiantes de primer semestre de un técnico auxiliar de una Institución en Bucaramanga. Se tuvo en cuenta como criterio de exclusión estudiantes de primer semestre mayores de 22 años. Los instrumentos de recolección de datos utilizados son dos encuestas sobre las estrategias didácticas de enseñanza y la formación en valores las cuales tienen su fundamentación teórica en dimensiones propias de estas dos variables de investigación. Resultados. se logra encontrar una correlación positiva moderada de 0.508 entre las estrategias didácticas de enseñanza y la formación en valores, con un nivel de significancia de $(\mathrm{p}=0.000)$ de los estudiantes de primer nivel. Conclusiones. Se considera importante abordar el tema planteado en esta investigación desde un enfoque cualitativo de manera complementaria, dadas las percepciones que pueden surgir desde los docentes y estudiantes en el proceso de enseñanza-aprendizaje. Las experiencias subjetivas de estas dos partes pueden construir una escala de valores distinta teniendo en cuenta los diferentes ámbitos sociales, familiares y educativos en los cuales se desarrollan diversas percepciones frente a la labor de enfermería.

Palabras claves:

Ética; Educación en Enfermería; Enseñanza; Valores Sociales; Formación del Profesorado; Cognición.

\begin{abstract}
Introduction. Healthcare is a human endeavor. It has essential and ethical considerations, and it seeks the wellbeing of others. As such, healthcare practices should be studied and analyzed, not only in the light of natural sciences, but also applying human sciences as well. This article intends to describe the relationship between learning and teaching strategies and formation of values in freshman students of the assistant nursing technical program from an educational institute in Bucaramanga. Methodology. This is a cross-sectional, quantitative, descriptive and correlational study, using a sample of 80 freshman students of the assistant nursing technical program from an educational institution in Bucaramanga. The eligibility criteria were freshman students 22 years and older. In order to collect data, two surveys on learning and teaching strategies and formation of values were administered. The theoretical basis for the surveys were dimensions specific to these two investigation variables. Results. There is a moderate positive correlation of 0.508 between teaching and learning strategies and formation of values, with a level of significance of $(\mathrm{p}=0.000)$ from freshman students. Conclusions. It is important to address the subject of this investigation from a qualitative standpoint and using a complementary approach, considering the perceptions of both faculty and students that arise during teaching and learning. Subjective experiences from both parties may create two different scales of values, considering different societal, family and educational environments in which different perceptions, regarding the nursing profession, are conceived.
\end{abstract}

Keywords:

Ethics; Nursing Education; Teaching; Social Values; Teacher Training; Cognition.

\title{
RESUMO
}

Introdução. O cuidado em saúde é uma empresa humana, cujo valor essencial é de natureza moral, orientado para o bem; então, as práticas de cuidados em saúde devem ser estudadas e orientadas não apenas pelas ciências naturais, mas também pelas ciências humanas aplicadas. $\mathrm{O}$ objetivo deste artigo é determinar a relação entre as estratégias didáticas de ensino e a formação de valores dos alunos do primeiro semestre de um curso técnico de auxiliar de enfermagem de uma instituição de Bucaramanga. Métodos. Corresponde a um estudo quantitativo, correlacional, descritivo e transversal, com uma amostra por conveniência de 80 alunos de primeiro semestre de um curso técnico de auxiliar de uma Instituição em Bucaramanga. Alunos do primeiro semestre acima de 22 anos foram excluídos do estudo. Os instrumentos de coleta de dados utilizados são dois questionários sobre as estratégias didáticas de ensino e a formação de valores, que tem fundamentação teórica nas dimensões próprias destas duas variáveis de pesquisa. Resultados. É possível encontrar uma correlação positiva moderada de 0,508 entre as estratégias didáticas de 
ensino e a formação de valores, com nível de significância de $(p=0,000)$ dos alunos do primeiro semestre. Conclusão. Considera-se importante abordar a questão levantada nesta pesquisa a partir de uma abordagem qualitativa de maneira complementar, dadas as percepções que podem surgir de professores e alunos no processo de ensino-aprendizagem. As experiências subjetivas destas duas partes podem construir uma escala diferente de valores, levando em consideração os distintos âmbitos sociais, familiares e educacionais nos quais desenvolvem-se diversas percepções sobre o trabalho de enfermagem

Palavras-chave:

Ética; Educação em Enfermagem; Ensino; Valores Sociais; Capacitação de Professores; Cognição.

\section{Introducción}

Desde la perspectiva internacional existen diferentes estudios que describen la problemática de falta de valores en los estudiantes y profesionales de la salud. Para algunos autores los valores poseen un carácter histórico concreto, formados bajo la influencia de un orden social determinado y cambian con el desarrollo de la sociedad, es allí donde el estudiante debe practicar autodisciplina, honestidad, fidelidad a sus pacientes, voluntad, respeto, honradez y perseverancia, de otra forma, las personas entrarían en crisis al enfrentarse a situaciones nuevas de acuerdo con los cambios de la sociedad (1).

En lo que respecta específicamente a los valores en los enfermeros y enfermeras, "el cuidado a la salud es una empresa humana, cuyo valor esencial es de índole moral, orientado hacia el bien, entonces las prácticas de cuidados de salud deberán ser estudiadas y orientadas no sólo por las ciencias naturales, sino por las ciencias humanas aplicadas" (2); por lo tanto, el autor hace énfasis en que, "tenemos un subsistema de educación media que crece desordenadamente capacitando enfermeras para asumir funciones de enorme complejidad en lo técnico y tecnológico, y en relación interhumana, con los pacientes y su familia, pues la calidad tiene una dimensión técnica y otra humana, la cual no se suple del todo con disposición humanitaria, como se ha comprobado en nuestra propia historia de cien años como profesión" (2).

Es por ello que se sabe que los errores, equivocaciones y las iatrogenias que suceden en enfermería, dependen de la capacidad técnica, pero mucho más, de la identidad, con la visión amplia de la función profesional que necesitan las personas y la sociedad a la que servimos, de la concentración en nuestro quehacer cotidiano, de la sobrecarga de tareas, pero también de la identidad con los valores que hemos heredado de quienes han hecho un gran esfuerzo para construir una Disciplina Científica y Humanista para el siglo XXI.
Un estudio realizado y documentado por Hernández, Bujardón, Iglesias y Seijo (3), titulado Estrategia educativa para la educación en valores humanos con métodos participativos en estudiantes de Enfermería, tiene por objetivo principal diseñar una estrategia educativa. En el documento se muestra la realización de un trabajo de educación en valores humanos con métodos participativos en estudiantes del nuevo modelo formativo de Enfermería de la Universidad de Ciencias Médicas de Camagüey en la Filial de Nuevitas, Cuba; como metodología se aplica una encuesta a estudiantes sobre valores, así como unas guías de orientación para evaluar otro grupo de valores, se implementan encuestas a docentes sobre los métodos participativos para la educación en valores. En los resultados principales se halla que se puede favorecer la educación en valores mediante métodos participativos, tales como responsabilidad, solidaridad, honestidad. Es importante implementar la estrategia dirigida a la preparación de profesores para que desarrollen métodos participativos y sus currículos, y se generen espacios de diálogo, reflexión y debate frente a las posturas pedagógicas.

En lo que respecta a casos latinoamericanos, en Brasil se percibe la enfermería como práctica social que simboliza entender sus dimensiones técnico-operativas, derivadas del esmero directo del conocimiento biotecnológico, y verla como ciencia práctica de la sociedad, con las cuales participa el compromiso por la salud. La enfermería es vista como parte fundamental de la producción de salud, guardando reciprocidad, por lo cual, los enfermeros necesitan ampliar una visión tolerante y participativa de las cuestiones sociales y de la salud, en consonancia con la complejidad de estas áreas y las pluralidades de la sociedad actual con la finalidad social del trabajo y de las instituciones sociales (4).

En Colombia, también se ha establecido diferentes contextos sobre la problemática de valores, es por ello que frente a un escenario de post conflicto, de violencia, de situación de orden público como lo vive el país, este genere espacios donde el personal encargado de atención de pacientes sea propicio para lograr la excelencia en la atención humana, ya que dicha labor de fortalecimiento 
en valores humanos exige, en las condiciones actuales, un profundo conocimiento acerca de las concepciones científicas de este problema, despojando a la práctica educativa de todo enfoque empirista y de espontaneidad que la ausencia de dominio profundo de este aspecto provoca.

Por su parte, el sistema de salud en Colombia y los profesionales del mismo sistema no han sido ajenos a la problemática del servicio de salud de América Latina, esto se debe a las reformas políticas en salud, a la perspectiva médico asistencial y a la concepción de seguridad social que desconoce las necesidades de salud de las poblaciones y la calidad de vida de las personas. Tanto en el contexto internacional como el nacional, se muestra la insatisfacción por el deterioro de las condiciones de trabajo y el aumento de la carga laboral, esta se debe a que las enfermeras y enfermeros han perdido garantías laborales en el sector por la escasa presencia de estos profesionales en las organizaciones gremiales, sindicales y de educación continua; por lo tanto, se requiere la participación del colectivo de enfermeras y de las agremiaciones para la garantía del ejercicio profesional y de la seguridad laboral (5).

Esto se debe a que el aprendizaje de la atención se establece en la relación entre el pacientey el enfermero(a), donde el paciente requiere que su atención sea humana, integral, ética, responsable y con conocimientos, por ello el enfermero pone en práctica las lecciones aprendidas en su formación, a través de acciones de cuidado, y buenas relaciones con los pacientes. Es allí que el papel del educador es fundamental, su actuación en este contexto se transforma en un elemento importante del proceso, ya que, al integrarse con los estudiantes en los campos clínicos, el docente proporciona, a través de su conocimiento personal y sus conocimientos actuales, las bases y el apoyo necesario para el desarrollo del futuro profesional de enfermería (5).

Por otra parte, la enfermería como profesión ha tenido un pasado perdurable que ha respondido a los cambios y desafíos dentro de un complejo sistema de salud. En una encuesta de Gallup en 2014, la enfermería fue reconocida como la mejor profesión en las áreas de honestidad y estándares éticos. Durante los últimos trece años, las enfermeras se han ganado este honor. Según la presidenta de la Asociación Estadounidense de Enfermeras (ANA), Dra. Pamela Cipriano, "el público pone su fe en las enfermeras para practicar éticamente. La salud, la autonomía e incluso la vida o la muerte del paciente pueden verse afectadas por las decisiones y acciones de la enfermera". Las enfermeras tienen un compromiso con el público ya que el público reconoce la capacidad de las enfermeras de cuidar a quienes buscan salud y curación (6).

La asociación Americana de Enfermeras estableció un código guía para el personal profesional en enfermería, cuyo objetivo era crear declaraciones normativas no negociables que describan las obligaciones, valores y principios para las enfermeras como individuos, grupos de enfermeras y la profesión. El Código proporciona el marco para que las enfermeras comprendan su compromiso con las personas, las familias, las comunidades y las poblaciones (2).

Desde sus inicios, el Código ha sido visto como una guía para el análisis ético y la toma de decisiones dentro de la profesión. Se basa en "la teoría, la práctica y la praxis de la enfermería en su expresión de los valores, virtudes y obligaciones que dan forma, guían e informan a la enfermería como una profesión" (6). El Código sirve como un recurso para las enfermeras en su entorno ético para ejecutar sus responsabilidades y obligaciones éticas.

Se espera que las enfermeras defiendan los valores e ideales de la profesión de enfermería en todas las áreas de su vida (6). Las personas que aspiran a ingresar en la profesión de enfermería necesitan orientación y educación adicional dentro del plan de estudios de la escuela de enfermería. Los educadores de enfermería tienen la oportunidad y la obligación de guiar a los estudiantes de enfermería de pregrado y posgrado en las áreas de ética de enfermería, análisis ético y toma de decisiones éticas para producir profesionales éticos.

Teniendo en cuenta lo anterior, es importante destacar que la falta de estrategias didácticas de enseñanza para la formación en valores a estudiantes de enfermería, influye en su nivel de participación activa y analítica, de las actuaciones con criterio ético. Así mismo, se plantea la necesidad de implementar estas estrategias con el fin de optimizar y mejorar la calidad de su labor, en su futuro profesional. La autonomía, responsabilidad, hábitos de trabajo en grupo, transdisciplinariedad y formación autónoma, deben ser los pilares de formación, con el fin de que su participación ética y cívica sea la directriz de una cultura de solidaridad y de servicio a la sociedad, comprometidos con las transformaciones que contribuyan a la justicia social y el desarrollo del país (7).

Por lo tanto, la educación en enfermería debe ser asumida como un trabajo en equipo, que permita utilizar y desarrollar recursos de aprendizaje de acuerdo a las exigencias actuales, sin dejar de un lado que se 
puede aprovechar la tecnología y la actividad científica a través de los medios que existen. Esto involucra, a su vez, la generación de ambientes adecuados de aprendizaje, donde se vincule el crecimiento personal con el desarrollo académico para fortalecer una formación basada en la crítica, la participación y los valores (8). Por consiguiente, los diseños curriculares actuales en la formación de los profesionales de enfermería precisan una revisión sistemática, profunda y actualizada de las necesidades en salud de la población. A fin de satisfacer estas necesidades, se debe desarrollar en el estudiante el empoderamiento como una forma visible de identidad profesional para que se facilite la adquisición de poder, autonomía y responsabilidad y, a su vez, se cumpla con las demandas de la sociedad y con la prestación de los servicios (8).

Lograr este cambio en el estudiante requiere que los profesionales responsables en la formación académica conozcan, implementen y evalúen, la aplicación de las diferentes estrategias pedagógicas. Durante mucho tiempo, el proceso de formación de los estudiantes de enfermería estuvo basado en la transmisión de contenidos del docente a los estudiantes de manera pasiva, sin involucrar motivación, intereses, experiencias de los estudiantes lo que no permitió el desarrollo del pensamiento crítico y de valores. El impacto de las estrategias tradicionales en la formación de enfermeros de una institución de Bucaramanga limita el aprendizaje y la capacidad de crítica de análisis, no hay motivación para la búsqueda de nuevos conocimientos, promueve una dificultad para relacionar e integrar los conocimientos obtenidos en las asignaturas y la puesta en práctica de la teoría, no desarrolla destrezas interpersonales para relacionarse con sus pacientes de manera ética.

De tal forma, que a partir de allí surge la necesidad de implementar nuevas estrategias pedagógicas para generar una formación complementaria en los estudiantes, en donde se establezca una relación con otras personas mediante el desarrollo de habilidades y destrezas interpersonales (9). Por este motivo, en este estudio se plantea la necesidad de proyectar estrategias didácticas de enseñanza para los estudiantes de primer semestre de Técnico Auxiliar en Enfermería de una institución de Bucaramanga, las cuales estén orientadas no solo a la adquisición de conocimientos propios del área de salud, sino que además incluyan estrategias para la formación de valores, con el propósito de fortalecer la formación integral de los estudiantes de enfermería. De esta forma, el objetivo principal de este artículo de investigación es determinar la relación entre las estrategias didácticas de enseñanza y la formación de valores de los estudiantes de primer semestre de Técnico Auxiliar en Enfermería de una Institución de Bucaramanga.

\section{Metodología}

La presente investigación es de enfoque cuantitativo, el cual "usa la recolección de datos para probar hipótesis, con base en la medición numérica y el análisis estadístico, para establecer patrones de comportamiento y probar teorías". Es de corte transversal y correlacional, lo que indica que recopila información en un momento único y determinado, "su propósito es describir variables y analizar su incidencia e interrelación en un momento dado" y es correlacional, puesto que estos estudios "tienen como finalidad conocer la relación o grado de asociación que exista entre dos o más conceptos, categorías o variables en un contexto en particular" (10).

La muestra ha sido seleccionada mediante el método no probabilístico por conveniencia, que permite "seleccionar aquellos casos accesibles que acepten ser incluidos" (8), aquellos casos a los que puede acceder el investigador para desarrollar su estudio. En este caso, la muestra estuvo conformada por 80 estudiantes de primer semestre de técnico laboral en Auxiliar de Enfermería de una institución de Bucaramanga. De los cuales 70 son mujeres entre los 17 y 22 años, y 10 hombres entre los 17 y 21 años. El estudio excluyó aquellos estudiantes que en el momento de la investigación estuvieran matriculados en el curso de primer semestre de enfermería con una edad mayor a 22 años.

La hipótesis principal consiste en confirmar si existe una relación significativa entre las estrategias didácticas de enseñanza y la formación en valores de los estudiantes de primer semestre de Técnico Auxiliar en Enfermería de una institución de Bucaramanga.

Para la medición de las variables se diseñaron dos instrumentos creados por la autora de la investigación y se aplicaron pruebas de fiabilidad mediante Alfa de Cronbach, a su vez, contó con la validación de tres expertos quienes hicieron correcciones a distintas preguntas teniendo en cuenta las dimensiones y los referentes teóricos propuestos para elaboración del instrumento, en términos de pertinencia, relevancia y claridad. En la tabla 1 se presenta la operacionalización de las variables.

La encuesta sobre las estrategias didácticas de enseñanza está compuesta por 30 preguntas tipo cerradas en una escala Likert de cinco puntos (Totalmente de acuerdo, de acuerdo, ni de acuerdo ni en desacuerdo, en desacuerdo, totalmente en desacuerdo). Se divide de la siguiente manera: 10 preguntas para evaluar la dimensión cognitiva, 10 preguntas para evaluar la dimensión cooperativa y 
Tabla 1. Operacionalización de las variables

\begin{tabular}{|c|c|c|c|c|c|c|}
\hline $\begin{array}{c}\text { Variable } \\
\mathbf{X}\end{array}$ & $\begin{array}{l}\text { Definición } \\
\text { conceptual }\end{array}$ & $\begin{array}{c}\text { Definición } \\
\text { operacional }\end{array}$ & Dimensiones & Indicadores & Nivel & Rango \\
\hline $\begin{array}{l}\text { Estrategias } \\
\text { Didácticas de } \\
\text { enseñanza }\end{array}$ & $\begin{array}{c}\text { Son } \\
\text { procedimientos } \\
\text { empleados por } \\
\text { el profesor para } \\
\text { hacer posible el } \\
\text { aprendizaje del } \\
\text { estudiante. } \\
\text { Incluyen } \\
\text { operaciones } \\
\text { físicas y mentales } \\
\text { para facilitar la } \\
\text { confrontación del } \\
\text { sujeto que aprende } \\
\text { con el objeto de } \\
\text { conocimiento, } \\
\text { considerados } \\
\text { como los } \\
\text { factores que } \\
\text { más contribuye } \\
\text { al progreso del } \\
\text { aprendizaje de } \\
\text { los estudiantes es } \\
\text { el grado y sobre } \\
\text { todo el modo en } \\
\text { que construyen } \\
\text { su conocimiento. } \\
\text { Para que las } \\
\text { estrategias de } \\
\text { aprendizaje se } \\
\text { asimilen y puedan } \\
\text { transferirse y } \\
\text { generalizarse } \\
\text { es preciso que } \\
\text { se enseñen y se } \\
\text { instrumenten } \\
\text { a través de las } \\
\text { diferentes áreas } \\
\text { curriculares, } \\
\text { 1997orre y } \\
\text { Rocabert, p.148, } \\
\text { 1997) }\end{array}$ & $\begin{array}{l}\text { Conjunto de } \\
\text { actividades } \\
\text { y técnicas de } \\
\text { enseñanza } \\
\text { escogidas por } \\
\text { el docente, las } \\
\text { cuales buscan } \\
\text { el logro de una } \\
\text { meta educativa, } \\
\text { establecida } \\
\text { previamente }\end{array}$ & $\begin{array}{l}\text { Estrategias } \\
\text { cooperativas }\end{array}$ & $\begin{array}{l}\text { Resumen. } \\
\text { Construcción de } \\
\text { mapas y gráficos. } \\
\text { Cuadro } \\
\text { sinóptico. } \\
\text { Método o } \\
\text { análisis de casos. } \\
\text { Discusión y } \\
\text { debate. } \\
\text { Análisis crítico y } \\
\text { reflexión. } \\
\\
\text { Trabajo en } \\
\text { equipo } \\
\text { Trabajo } \\
\text { cooperativo } \\
\text { Destrezas y } \\
\text { habilidades } \\
\text { Cormación del } \\
\text { Cuidado de la } \\
\text { salud mental. } \\
\text { Diferencias } \\
\text { individuales. } \\
\text { Conductas } \\
\text { saludables. } \\
\text { Reflexión } \\
\text { Apoyo grupal } \\
\text { Conociomiento de } \\
\text { del otro } \\
\end{array}$ & $\begin{array}{l}\text { Excelente } \\
\text { Bueno } \\
\text { Regular } \\
\text { Malo } \\
\text { Deficiente } \\
\\
\\
\text { Excelente } \\
\text { Bueno } \\
\text { Regular } \\
\text { Malo } \\
\text { Deficiente }\end{array}$ & 更 \\
\hline
\end{tabular}

Fuente: Autora 
10 preguntas para evaluar la dimensión axiológica. La encuesta sobre la formación en valores está compuesta por 15 preguntas tipo cerradas con la escala Likert igual que la anterior encuesta. Se divide de la siguiente manera: 5 preguntas para evaluar los referentes éticos, 5 preguntas para evaluar los juicios éticos y 5 preguntas para evaluar la autenticidad. Según las respuestas dadas en cada dimensión se determina cuáles estrategias didácticas de enseñanza se están brindando y cómo es la formación en valores.

El proceso para el análisis estadístico de los datos se realiza de acuerdo con las siguientes fases (10):

- Selección del software: IBM SPSS Statistics versión 2.3

- Ejecución del programa: categorizar numéricamente cada una de las respuestas para ejecutar el programa.

- Análisis de datos: datos descriptivos por cada variables y datos correlacionales de las dimensiones de estrategias de enseñanza y la formación en valores.

- Preparación de resultados: presentación de gráficas y tablas según corresponda.

- Interpretación de resultados: interpretar los resultados a partir de lo evidenciado en las gráficas y tablas.

Figura 1. Gráfico de dispersión entre estrategias didácticas y formación en valores.

Gráfico de dispersión entre estrategias didácticas y formación en valores

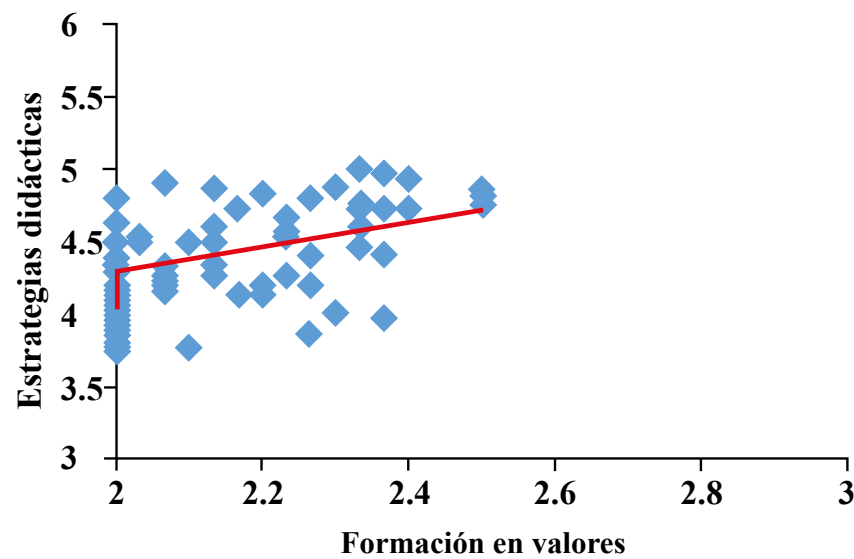

Fuente: Elaboración propia.
Este estudio se rige bajo las normas éticas para la investigación de acuerdo con la Resolución 008430 de 1993 del Ministerio de Salud, por la cual se establecen las normas científicas, técnicas y administrativas para la investigación en salud (11).

\section{Resultados}

\section{Correlación entre estrategias didácticas y formación en valores (Figura 1)}

De acuerdo con los resultados obtenidos en la correlación entre la dimensión cognitiva y los referentes éticos, existe una correlación positiva y moderada de 0.508 , estadísticamente significativa $(\mathrm{p}=0.000)$. Esto indica que a medida que una variable aumenta la otra variable también aumenta, es decir que si las estrategias didácticas de enseñanza son efectivas en los estudiantes su formación en valores también.

\section{Correlación entre estrategias cognitivas y formación en valores (Figura 2)}

De acuerdo con los resultados obtenidos en la correlación entre la dimensión cognitiva y formación en valores, existe una correlación positiva y moderada de 0.449 , estadísticamente significativa $(p=0.000)$. Esto indica que a medida que una variable aumenta la otra variable también aumenta, es decir que si las estrategias cognitivas son efectivas en los estudiantes su autenticidad también.

Figura 2. Gráfico de dispersión entre dimensión cognitiva y Formación en valore.

\section{Gráfico de dispersión entre dimensión cognitiva y Formación en valores}

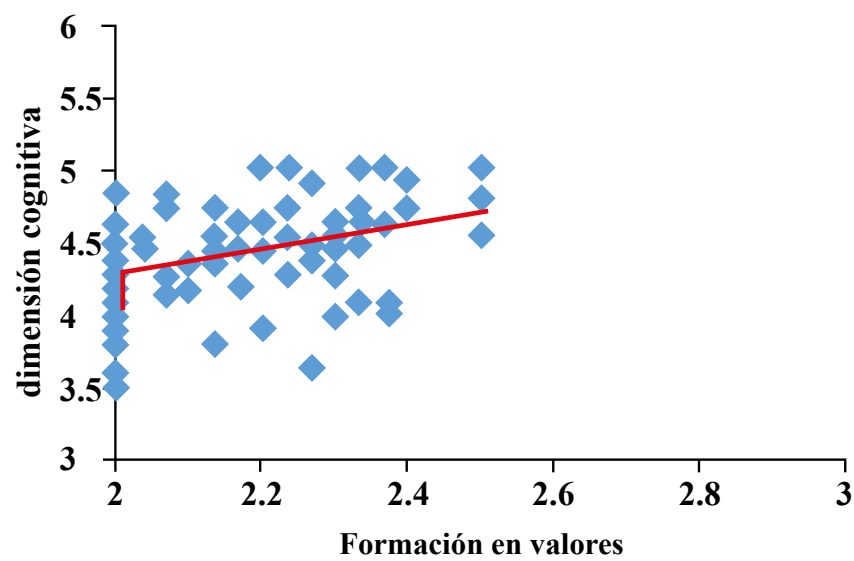

Fuente: Elaboración propia. 


\section{Correlación entre estrategias cooperativas y forma- ción en valores (Figura 3)}

De acuerdo con los resultados obtenidos en la correlación entre la dimensión cooperativa y formación en valores, existe una correlación positiva y baja de 0.386, estadísticamente significativa $(\mathrm{p}=0.000)$. Esto indica que a medida que una variable aumenta la otra variable también aumenta, es decir que si las estrategias cooperativas son efectivas en los estudiantes su autenticidad también.

Figura 3. Gráfico de dispersión entre dimensión cooperativa y Formación en valores.

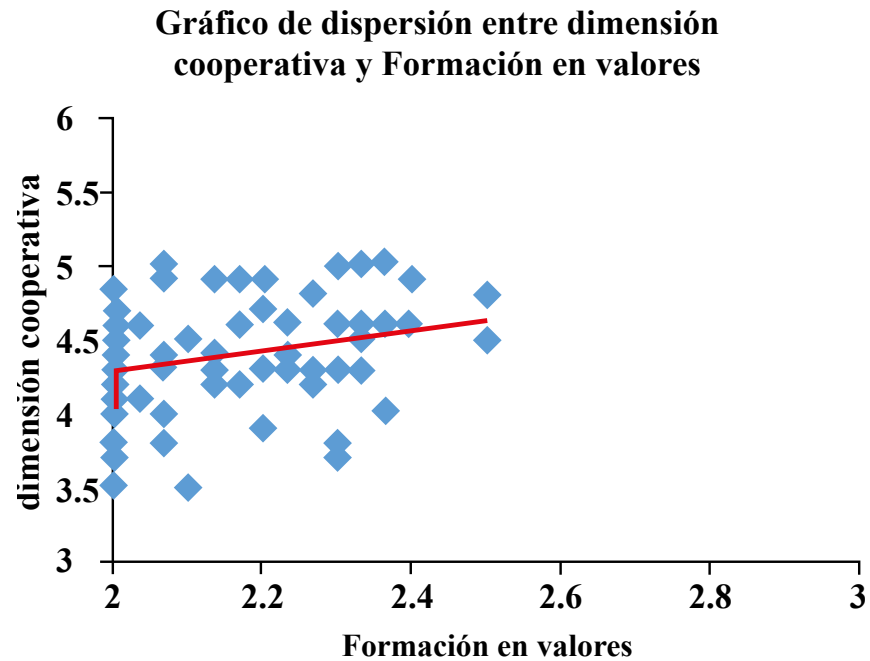

Fuente: Elaboración propia.

\section{Discusión}

De acuerdo con los resultados obtenidos a partir de los análisis estadísticos descriptivos y los correlacionales sobre las estrategias didácticas de enseñanza y la formación en valores en los estudiantes de primer nivel de Técnico en Auxiliar de Enfermería de una institución de Bucaramanga; se procede a dar cumplimiento al objetivo principal, determinar la relación entre las estrategias didácticas de enseñanza y la formación de valores de los estudiantes de primer semestre de Técnico Auxiliar en Enfermería de una institución de Bucaramanga; y a constatar las hipótesis de investigación con la información recopilada en los antecedentes investigativos.

Frente a la hipótesis principal se encontró que, de acuerdo con el coeficiente de Pearson, existe una correlación positiva moderada de 0.508 y significativa $(p=0.000)$

\section{Correlación entre estrategias axiológicas y forma- ción en valores (Figura 4)}

De acuerdo con los resultados obtenidos en la correlación entre la dimensión axiológica y formación en valores, existe una correlación positiva y moderada de 0.441 , estadísticamente significativa $(p=0.000)$. Esto indica que a medida que una variable aumenta la otra variable también aumenta, es decir que si las estrategias axiológicas son efectivas en los estudiantes su autenticidad también.

Figura 4. Gráfico de dispersión entre dimensión axiológica y Formación en valores.

\section{Gráfico de dispersión entre dimensión axiológica y Formación en valores}

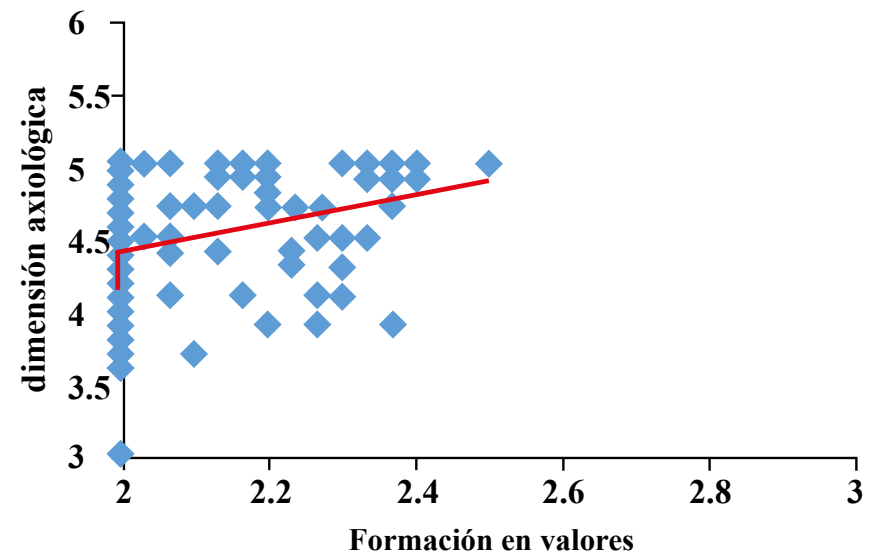

Fuente: Elaboración propia.

entre las estrategias didácticas de enseñanza y la formación en valores; comprobando así que se mantiene la hipótesis formulada inicialmente. De forma similar, en el trabajo titulado "Enseñanza de estrategias de aprendizaje en el aula. Estudio descriptivo en profesorado de niveles no universitarios" se realiza una investigación no experimental, transversal, exploratoria, descriptiva y correlacional, en la cual se trabajan ocho objetivos relacionados con las estrategias de enseñanza y aprendizaje con el fin de conocer el método de enseñanza en las aulas; y de otro lado, buscar la manera de mejorarlo, de manera que los estudiantes puedan regular su propio proceso de aprendizaje de manera autónoma (12). Al trabajar el sexto objetivo, establecer si existía o no relación entre la enseñanza de estrategias y factores del centro como tipo de enseñanza, etapa y metodología utilizada por parte del docente; se calcularon los coeficientes de asociación Eta2 entre cada una de las variables del centro y la puntuación global obtenida del 
cuestionario. De otro lado, para comprobar si las diferencias encontradas en la puntuación general obtenida son estadísticamente significativas, se utilizó la prueba $\mathrm{T}$ de Student $\mathrm{y}$, finalmente, mediante la prueba de Kruskal-Wallis, ANOVA, se trabajaron los dados que no cumplen criterios de normalidad (12).

El análisis de los resultados arrojó que las variables de centro como la provincia y el tipo de enseñanza ya sea mixta o diferenciada no tienen ningún efecto en la enseñanza de estrategias de aprendizaje, con valores de Eta2 de 0.0004 y 0 respectivamente. Por otra parte, el tipo de centro tiene un efecto pequeño pero significativo en el uso y enseñanza de estrategias de recuperación y transferencia, y de estrategias motivacionales, con valores Eta2 de 0.0126 y 0.0093 . La metodología del docente que se emplea afecta al uso de las estrategias de atención, motivación, metacognitivas y de autorregulación (12).

Respecto al propósito de conocer si existía una relación entre las estrategias de aprendizaje utilizadas en el aula y la materia que se impartía, se calculó el coeficiente de asociación Eta2 y, de acuerdo con los resultados, se encontró que existe una relación que, aunque pequeña, es considerada significativa entre la materia que se imparte y el uso de estrategias de recuperación y transferencia y de estrategias motivacionales, con valores Eta2 de 0.0199 y 0.0219 respectivamente (12).

Por su parte, en la tesis titulada "percepción de docentes, estudiantes de la carrera de enfermería y usuarios del hospital "José Garcés Rodríguez" de salinas sobre la aplicación de la ética en el desempeño profesional 2011 -2012”, se encuentra que la formación que se ofrece en la carrera de enfermería para la formación profesional se basa fundamentalmente en el aprendizaje de teorías relacionadas con la enfermería, en la calidad de la atención, en fomentar los valores humanos para que se pueda dar una atención a los usuarios con calidad y calidez, haciendo énfasis en la responsabilidad en el campo laboral en un $74 \%$, en la seguridad y autodominio en un $13 \%$ cada uno (13).

En cuanto a la confirmación de hipótesis se encontró que, de acuerdo con el coeficiente de Pearson, existe una correlación positiva moderada de 0.449 y significativa $(p=0.000)$ entre las estrategias cognitivas y la formación en valores, comprobando así que se mantiene la hipótesis específica formulada inicialmente. De manera similar, en el estudio titulado "Motivación, estrategias de aprendizaje y rendimiento académico en estudiantes de ESO", se plantea una correlación entre las estrategias cognitivas y las metas de aprendizaje propuestas por los docentes $(p<0.05)$ en donde se halla que entre más altas son las metas de aprendizaje mayor es el uso de los estudiantes de las estrategias cognitivas, y cuanto más bajas son las metas, menor es el uso de estrategias cognitivas; en este estudio las metas de aprendizaje son enfocadas al desarrollo de competencias propias de la profesión (14).

En relación con las correlaciones, de acuerdo con el coeficiente de Pearson, existe una correlación positiva baja de 0.386 y significativa $(p=0.000)$ entre las estrategias cooperativas y la formación en valores; comprobando así que se mantiene la hipótesis específica formulada inicialmente. De forma contraria, en el artículo titulado "Impacto de la educación basada en competencias en el aprendizaje de alumnos de octavo semestre de licenciatura en enfermería en una universidad pública", se halla que de acuerdo con las estrategias utilizadas por el docente se favoreció el aprendizaje en un $32.1 \%$; sin embargo, las actividades realizadas en trabajo en equipo solo se vieron favorecidas en un $48.2 \%$; y que un porcentaje de $7.1 \%$ de los estudiantes no les gusta trabajar en equipo, lo que no permite afianzar las relaciones de los estudiantes de manera cooperativa con los demás compañeros (15).

La correlación entre las estrategias axiológicas y la formación en valores fue positiva moderada $(0.441)$ y significativa $(\mathrm{p}=0.000)$; comprobando así que se mantiene la hipótesis específica formulada inicialmente. En el mismo estudio (15), se encontró que el 50\% de los alumnos percibió que aprendieron valores profesionales de enfermería en un nivel bueno, y el $32.1 \%$ en un nivel excelente. Entre esos valores que se resaltaron durante $\mathrm{su}$ formación fueron servicio $(26.7 \%)$, solidaridad (26.7\%), responsabilidad (28.5\%) y empatía (17.8\%).

Contrario a esto, en la investigación titulada "La pérdida de valores en estudiantes de enfermería identificados por sus profesores", se halla que las manifestaciones que evidencian la pérdida de valores en estudiantes, y reflejadas por los profesores, cuyas estrategias axiológicas no son enseñadas de manera general son la falta de profesionalismo en $92.3 \%$, presencia de indisciplina en un $88.4 \%$, la falta de educación en un $84.6 \%$, otras menos representadas son la falta de sentido de pertenecía y poca dedicación a lo que están estudiando con un 76.9 y $61.5 \%$ respectivamente (1).

En lo que respecta a los antecedentes aportados en la investigación, la mayoría de ellos tuvieron un enfoque cualitativo, lo que no permite realizar comparativos con esta investigación en relación con los análisis estadísticos realizados. Sin embargo, algunas investigaciones han mostrado que existe una relación entre la formación de 
valores y el desarrollo de competencias profesionales de los auxiliares en enfermería, en donde se muestra que las estrategias didácticas de enseñanza no deben ser únicamente procesos instructivos y de aplicación de normativas pedagógicas, sino también procesos metodológicos con enfoques científicos y sociales $(3,6,16)$; es decir, procesos en los cuales además de brindar formaciones intelectuales, también sirvan para formar estudiantes con valores que sean capaces de entender la realidad cotidiana, social y ambiental en la cual se desenvuelven de una forma ética y socialmente responsable (16). Así como quienes sostienen que se deben sacar adelante los modelos estratégicos de enseñanza en la formación en valores, teniendo en cuenta la importancia de formar personas líderes que promuevan la sana convivencia social y la superación personal de cada uno de los involucrados en los procesos educativos (17). Sin embargo, las pocas investigaciones cuantitativas encontradas en este estudio muestran la necesidad de seguir abordando este tema desde metodologías cualitativas, dado que los resultados encontrados en esta investigación solo proporcionan un enfoque parcial frente a la relación entre las estrategias de enseñanza y la formación en valores de estudiantes de enfermería.

\section{Conclusiones}

Con respecto al objetivo general de la investigación, determinar la relación entre las estrategias didácticas de enseñanza y la formación de valores de los estudiantes de primer semestre de Técnico Auxiliar de Enfermería de una institución de Bucaramanga; los resultados indican que existe una correlación positiva moderada de 0.508 según el coeficiente de Pearson y una significancia de $(\mathrm{p}=0.000)$ entre las estrategias didácticas de enseñanza y la formación en valores. Por lo tanto, se mantiene la (Hp): Existe una relación significativa entre las estrategias didácticas de enseñanza y la formación en valores de los estudiantes de primer semestre de Técnico Auxiliar en Enfermería de una institución de Bucaramanga.

Así, dado el desarrollo de esta investigación de naturaleza cuantitativa, se considera importante abordar el tema planteado en esta investigación desde un enfoque cualitativo de manera complementaria, dadas las percepciones que pueden surgir desde los docentes y estudiantes en el proceso de enseñanza-aprendizaje. Las experiencias subjetivas de estas dos partes pueden construir una escala de valores distinta, teniendo en cuenta a los diferentes ámbitos sociales, familiares y educativos en los cuales se desarrollan diversas percepciones frente a la labor de enfermería. Con este estudio se logra concluir desde una metodología cuantitativa que las estrategias didácticas de enseñanza se relacionan con la formación en valores; sin embargo, es relevante ver cómo la formación valores es asumida desde el aprendizaje de cada uno de los estudiantes en los diferentes niveles de formación.

Dada la falta de un enfoque cualitativo en esta investigación, se observa una limitación en un estudio con un diseño único, de esta manera, se debe realizar un estudio cualitativo complementario para obtener resultados objetivos de esta investigación. Como se observó en las investigaciones encontradas, existen falencias en la formación de enfermeros en la parte ética, lo que repercute en el desempeño de sus labores, y es por esto que las futuras líneas de investigación deben estar centradas en la parte educativa de la labor de enfermería dado que los conocimientos obtenidos por los estudiantes no pueden limitarse al campo de salud, sino que deben incidir en aspectos del ser humano para garantizar una formación integral.

Asimismo, se resalta la idea de seguir fortaleciendo las estrategias didácticas de enseñanza a través de actividades relacionadas con los componentes cognitivos, cooperativos y axiológicos que fortalecen, a su vez, la formación en valores de los estudiantes de enfermería, y de manera complementaria generar programas educativos transversales basados en competencias intrapersonales e interpersonales.

Dentro de las limitaciones del estudio se considera que los participantes que se encontraban en su primer semestre de formación, es posible que no hayan logrado incorporar todas las herramientas recibidas sobre la formación en valores, por tal motivo se considera apropiado que se realicen más estudios en semestres avanzados, combinando diseños de investigación cuantitativa y cualitativa que permitan identificar las percepciones de los estudiantes sobre el aprendizaje realizado en el aula, sobre los contenidos que desarrolla y, al mismo tiempo, percepciones del docente sobre la temática de los valores y sobre la estrategia de enseñanza con la cual siente que logra afianzar, apropiar o cambiar (si es necesario dentro de su proceso de reflexión personal).

\section{Referencias}

1. Gómez R, Ramos D, Sánchez J, Rodríguez L. La pérdida de valores en estudiantes de enfermería identificados por sus profesores. 2018;3(2015):110. 
2. Rubio Domínguez S. Principios y valores que guían el ejercicio del personal de enfermería. Rev Conamed. 2008;13:22-6.

3. Hernández M, Bujardón A, Iglesias N, Seijo B. Estrategia educativa para la educación en valores humanos con métodos participativos en estudiantes de Enfermería. Humanidades Médicas. 2013;13(1):224-43.

4. Lourdes E, Pavone C, Schveitzer MC. Valores de la enfermería como práctica social : una metasíntesis cualitativa. Rev Latinoam Enferm. 2013;21(3):1-9.

5. Zapata M. Enfermeria ¿Una Profesion En Crisis? El caso en la ciudad de Medellín - Colombia [Internet]. Universidad de Antioquia. 2008. Disponible en: http://bibliotecadigital.udea.edu.co/dspace/ handle/10495/169

6. Mora L. Los principios éticos y bioéticos aplicados a la calidad de la atención en enfermería. Rev Cuba Oftalmol. 2015;28(2):228-223.

7. Diaz M. Nivel de satisfacción de los estudiantes de Enfermería sobre la enseñanza en la asignatura Enfermería en salud del adulto y anciano de la E.A.P.E. de la UNMSM . [Internet]. Universidad Nacional Mayor de San Marcos; 2013. Disponible en: http:// cybertesis.unmsm.edu.pe/bitstream/handle/cybertesis/3530/Diaz gm.pdf;jsessionid=46C059D90D4DB134644B3F4FE60A034E? sequence $=1$

8. Salinas A, López JP. Magnificación: una nueva estrategia didáctica en Educación odontológica. Rev Dent Chile. 2015;106(3):21-4.

9. Achury Saldaña M. Estrategias pedagógicas en la formación de profesionales de enfermería. Investig en Enfermería Imagen y Desarro. 2008;10(2):97113.

10. Hernández R, Fernández C, Baptista P. Metodología de la investigación [Internet]. Vol. 53, Mc Graw Hill. 2014. 1-589 p. Disponible en: https:// mail.google.com/mail/u/1/\#inbox/15a4cf4b02ab7f85?projector $=1$

11. Ministerio de Salud. Resolución 008430 de 1993. Por la cual se establecen las normas científicas, técnicas y administrativas para la investigación en salud. Biomédica. 1993.

12. Javaloyes Sáez MJ. Enseñanza de estrategias de aprendizaje en el aula. Estudio descriptivo en profesorado de niveles no universitarios [Internet]. Valladolid; 2016. Disponible en: https://uvadoc.uva. es/bitstream/10324/16867/1/Tesis1021-160505.pdf

13. Pozo LA. Percepción de docentes, estudiantes de la carrera de enfermería y usuarios del hospital "José Garcés Rodríguez” de salinas sobre la aplicación de la ética en el desempeño profesional $2011-2012$. Universidad Estatal Península de Santa Elena; 2012.
14. Rodríguez G. Motivación, estrategias de aprendizaje y rendimiento académico en estudiantes de ESO [Internet]. A Coruña; 2009. Disponible en: http:// ruc.udc.es/dspace/bitstream/handle/2183/5669/RodriguezFuentes Gustavo TD 2009.pdf?sequence $=1 \&$ is Allowed $=y$

15. Pecina RM. Impacto de la educación basada en competencias en el aprendizaje de alumnos de octavo semestre de licenciatura en enfermería en una universidad pública. Rev Iberoam para la Investig y el Desarro Educ. 2013;10:1-20.

16. Bravo Mancero P, Varguillas Carmona CS. Estrategias didácticas para la enseñanza de la asignatura Técnicas de Estudio en la Universidad Nacional de Chimborazo. Sophía [Internet]. 2015;1(19):271. Disponible en: http://revistas.ups.edu.ec/index.php/ sophia/article/view/19.2015.13

17. Beltrán D, Romero G, Muñoz M. Estrategias lúdico-pedagógicas para fomentar una educación en valores para la sana convivencia del grado primero de básica primaria de la institución educativa Playas de Acapulco de Cartagena. Universidad de Cartagena; 2015. 